\title{
Exploração sexual de crianças e adolescentes em situação de rua no Sul do Brasil
}

\author{
Sexual exploitation of homeless children and adolescents \\ living on the streets in Southern Brazil
}

Lauro Miranda Demenech (https://orcid.org/0000-0002-7285-2566) 1,2

Simone dos Santos Paludo (https://orcid.org/0000-0002-3281-5824) ${ }^{3}$

Priscilla dos Santos da Silva (https://orcid.org/0000-0002-3125-9854) ${ }^{1}$

Alice Monte Negro de Paiva (https://orcid.org/0000-0002-4033-7826) ${ }^{1}$

Fernanda Fontes (https://orcid.org/0000-0002-3641-549X) ${ }^{1}$

Lucas Neiva-Silva (https://orcid.org/0000-0002-7526-2238) 1,3

${ }^{1}$ Centro de Estudos sobre Risco e Saúde (CERIS), Universidade Federal do Rio Grande (FURG). Av. Itália, km 8, Carreiros. 96203-900 Rio Grande RS Brasil. lauro_demenech@

hotmail.com

${ }^{2}$ Programa de Pós-

Graduação em Ciências da Saúde, FURG. Rio Grande RS Brasil.

${ }^{3}$ Programa de PósGraduação em Psicologia, FURG. Rio Grande RS Brasil.

\begin{abstract}
The scope of this study was to investigate the prevalence of sexual exploitation of homeless children and adolescents living on the streets, as well as characteristics associated with this outcome. In this cross-sectional investigation, carried out in the municipalities of Rio Grande and Porto Alegre-RS, data were collected through interviews, which were conducted by trained interviewers. The Respondent-Driven Sampling (RDS) strategy was adopted and statistical analyses were conducted using Poisson regression with robust adjustment of variance. The sample included 186 children and adolescents between 10 and 17 years of age, of which $8 \%$ reported having been subjected to sexual exploitation. Being 16 to 17 years old, having had forced sexual intercourse and not having bonds with school were characteristics independently associated with this outcome. It was observed that the lack of contact with school increased the probability 16-fold of having suffered sexual exploitation, being considered the main risk factor in this study. The proposition is that the inclusion and permanence of homeless children and adolescents living on the streets into schools might significantly reduce the risk of sexual exploitation of this population group.
\end{abstract}

Key words Homeless youths living on the streets, Sexual abuse of children and adolescents, School
Resumo O objetivo do estudo foi investigar a prevalência de exploração sexual entre crianças e adolescentes em situação de rua e as características associadas a este desfecho. Neste estudo transversal, conduzido nos municípios de Rio Grande e Porto Alegre-RS, os dados foram coletados por meio de entrevistas com os participantes, as quais foram conduzidas por entrevistadores treinados. O método de amostragem foi o Respondent-Driven Sampling (RDS) e as análises estatísticas foram conduzidas por meio da regressão de Poisson com ajuste robusto da variância. A amostra foi composta por 186 crianças e adolescentes com idades entre 10 e 17 anos, das quais $8 \%$ relataram ter tido experiência de exploração sexual. Ter idade entre 16 e 17 anos, ter sofrido relação sexual forçada e não ter vínculo escolar foram características independentemente associadas a este desfecho. Foi observada que a falta de vínculo escolar aumentou em 16 vezes a probabilidade de sofrer exploração sexual, sendo o principal fator de risco identificado neste estudo. Sugere-se que a inclusão e permanência das crianças e adolescentes em situação de rua no contexto escolar possa reduzir significativamente o risco de exploração sexual entre esta população.

Palavras-chave Jovens em situação de rua, Abuso sexual de crianças e adolescentes, Escola 


\section{Introdução}

A exploração sexual tende a causar marcas profundas e danos significativos no desenvolvimento daqueles que vivem em algum momento essa violência ${ }^{1-3}$. Configura-se como a mercantilização dos corpos de crianças e adolescentes para fins sexuais, tendo como objetivo a obtenção de lucro ou benefícios para o mediador e/ou aliciador. A terminologia vem sofrendo profundas mudanças que refletem dilemas éticos e tensionamentos sobre os papeis dos diferentes atores envolvidos ${ }^{4}$. Por esse motivo, ao tratar de exploração sexual comercial de crianças e adolescentes estão incluídas exploração sexual no contexto do turismo, pornografia infantil, tráfico de crianças e adolescentes para fins sexuais e exploração sexual comercial ${ }^{2}$. Esse conceito passou a ser usado no lugar do termo "prostituição" para evitar a culpabilização daqueles que são explorados e para destacar a existência de um agressor e de uma violação ${ }^{5}$. A exploração difere de outras formas de violência sexual devido à presença de moeda de troca, podendo ser dinheiro, presentes, comida, drogas, proteção ou não agressão $0^{1,6,7}$.

No ano de 2002, a International Labor Organization estimou que 1,8 milhões de crianças e adolescentes no mundo foram explorados sexualmente. Destes, 750 mil estavam na América Latina e Caribe ${ }^{8}$. Em 2006, o cenário tornou-se ainda mais alarmante, após a UNICEF reportar que três milhões de crianças e adolescentes estavam no contexto da exploração sexual ${ }^{9}$. Dados divulgados pela Childhood Brasil ${ }^{10}$ estimaram que cerca de 500 mil são vítimas da exploração sexual no país, tendo a maioria delas entre 7 e 14 anos de idade.

Apesar de não ser um fator determinante, crianças e adolescentes que vivem em contextos de violência e vulnerabilidade estão mais expostos à exploração sexual, pois os adultos que ali se encontram aproveitam-se da imaturidade emocional para explorá-los e tratá-los como mercadoria sexual ${ }^{11}$. Estar em situação de rua torna-os ainda mais suscetíveis à violação de direitos, devido a fatores como o enfraquecimento dos vínculos familiares, a ausência escolar, o trabalho infantil, o envolvimento com o tráfico de drogas, a exploração sexual e a inadequada proteção do Estado $^{12}$.

As iniquidades sociais desse grupo não se iniciam com a chegada no contexto da rua, a vida destas crianças e adolescentes envolve um processo dinâmico articulado aos aspectos sociais, econômicos, culturais e históricos vivenciados tam- bém por suas famílias, revelando os mais diversos processos de vulnerabilidade social e desfiliação, como referem alguns autores ${ }^{13}$.

No âmbito familiar, a presença da violência, vista em abusos e negligência, pode ser uma das principais causas que levam as crianças e os adolescentes a buscarem a rua ${ }^{12}$. O afastamento de casa parece ser a solução para a interrupção da violência familiar. Contudo, a rua rapidamente proporciona novas vitimizações, principalmente sexuais.

Abordar a exploração do corpo de crianças e adolescentes ainda é uma tarefa delicada, o que reforça a necessidade de dar visibilidade à temática com o intuito de contribuir para o preenchimento de lacunas na literatura científica, cooperando para o enfrentamento do problema e visando ampliar a garantia de direitos. Embora existam várias políticas setoriais de saúde que visam a identificação, o monitoramento e a contenção das situações de violência ${ }^{14}$, a exploração sexual de crianças e adolescentes em situação de rua pode escapar dos registros oficiais diante da própria dinâmica velada do crime cometido por adultos e da iniquidade social desse grupo. A produção de dados capaz de retratar o processo de vulnerabilidade e sua dinamicidade pode contribuir com o aprimoramento de ações integradas e intersetoriais no campo da saúde coletiva ${ }^{13}$. Dessa forma, o objetivo deste estudo foi investigar a prevalência de exploração sexual entre crianças e adolescentes em situação de rua, bem como investigar seus fatores associados.

\section{Método}

\section{Delineamento e participantes}

Este é um estudo quantitativo, com delineamento transversal, conduzido com crianças e adolescentes em situação de rua, com idades entre 10 e 17 anos, realizado nas cidades de Porto Alegre e Rio Grande, Rio Grande do Sul, Brasil. As faixas-etárias foram divididas de acordo com o Estatuto da Criança e do Adolescente, onde são considerados crianças os indivíduos de até 12 anos de idade incompletos, e adolescentes aqueles entre 12 anos completos e 18 anos incompletos $^{15}$. A situação de rua foi caracterizada como: passar parte do tempo diário no espaço da rua; realizar atividades no contexto da rua, incluindo trabalhar informalmente, brincar e perambular; e estar desacompanhado de um adulto responsável ${ }^{16-18}$. Esta pesquisa foi parte de um estudo 
maior que investigou as condições de saúde e vulnerabilidade de crianças, adolescentes e jovens em situação de rua nas cidades de Porto Alegre e Rio Grande-RS.

\section{Amostragem, cálculo amostral e procedimentos}

O método de amostragem deste estudo foi o Respondent-Driven Sampling (RDS) ou Amostragem Conduzida pelo Participante. Através do método "bola de neve", o RDS "pesa" a amostra para compensar o fato de que esta foi coletada, inicialmente, de uma maneira não-randômica. Este modelo pressupõe que as populações de difícil acesso (hard-to-reach populations) sejam recrutadas por seus próprios pares. O RDS é definido por meio de duas características principais: 1) não existe uma estrutura amostral definida, em que tanto o número de indivíduos quanto às fronteiras divisórias da população são desconhecidos; e 2) existe uma forte preocupação em relação à privacidade, principalmente porque os membros destas populações geralmente são estigmatizados ou estão associados a comportamentos ilícitos, o que leva os indivíduos a se recusarem a participar das pesquisas ou a fornecer respostas não-confiáveis ${ }^{19}$. É possível iniciar a seleção da amostra por um processo não probabilístico, tendo a possibilidade de alcançar uma amostra final probabilística através do método RDS. As análises são realizadas por meio do software RDSAT, que permite avaliar a amostra final alcançada e definir se a mesma alcançou características de uma amostra probabilística ${ }^{20}$. Essas características são contempladas quando a amostra se torna estável, alcançando o chamado "equilíbrio"19,20.

O processo amostral iniciou através da identificação das sementes, como foram chamadas as crianças e adolescentes. A seleção das sementes ocorreu de forma não-aleatória, na tentativa de representar a diversidade social e geográfica da população. Foram escolhidas, com a ajuda de organizações governamentais (OGs) e não-governamentais (ONGs), pessoas com uma intensa rede social, de ambas as cidades, as quais ficaram responsáveis pelo recrutamento dos próximos participantes. Cada participante-semente recebeu dois cupons-convite (numerados com código específico que possibilitou a organização sequencial de recrutamento), que seriam distribuídos a outros dois conhecidos, que também estivessem em situação de rua, os quais compuseram a primeira onda. Cada um destes sujeitos, depois de serem entrevistados, receberam dois outros cupons, que foram entregues aos novos participantes da terceira onda; e assim sucessivamente. Cada semente recebeu um ressarcimento, na forma de vale-alimentação, pela entrevista realizada e outro por cada participante convidado após também ter sido entrevistado. O método RDS pressupõe que o fato de ser a própria criança/adolescente quem convida o próximo participante tende a reduzir as "respostas socialmente aceitas", pois há menor desconfiança em relação a um convite feito pelo pesquisador. Além disso, são recrutados participantes aos quais os pesquisadores, de fato, não teriam acesso.

Em função do projeto maior investigar diferentes temáticas, foi realizado um cálculo amostral descritivo, usando como desfecho exploração sexual, com prevalência esperada de $10 \%$. Apesar de não existir um marco de amostragem definido, foi utilizado como referência a estimativa de 450 adolescentes e jovens em situação de rua no município de Porto Alegre ${ }^{21}$. Os parâmetros utilizados foram intervalo de confiança de 95\%, com margem de erro de 3 pontos percentuais e poder de $80 \%$. Ao total ainda foi acrescido $10 \%$, para compensar possíveis desistências. Com base nisso, estimou-se uma amostra de 207 participantes em Porto Alegre. Como não havia estimativa da população de crianças e adolescentes em situação de rua em Rio Grande, foi adotado como critério o valor relativo a um terço da amostra de Porto Alegre, correspondendo a 69 indivíduos para Rio Grande. Portanto, a amostra total do estudo deveria ter, no mínimo, 276 participantes. Assim, foram planejadas cinco sementes em Porto Alegre e duas sementes em Rio Grande, estimando-se cinco ondas por semente e $30 \%$ de interrupções na sequência de recrutamento. Devido a estas interrupções, ao longo da coleta de dados, foram inseridas duas novas sementes em Porto Alegre, perfazendo um total de sete sementes nesta cidade.

\section{Instrumentos e variáveis}

Com cada participante foi realizada uma entrevista, cuja estrutura foi elaborada a partir de instrumentos utilizados em outros estudos com essa população ${ }^{17,21}$. Todos os entrevistadores receberam treinamento sobre técnicas de entrevista e aspectos éticos com populações de risco. O trabalho de campo foi realizado sempre em duplas ou trios e as entrevistas aconteceram em locais abertos, como praças e parques, de ampla circulação da população-alvo. Para as entrevistas, 
foram eleitos lugares com menor exposição e/ou trânsito de pessoas, a fim de preservar a integridade do participante e a qualidade das informações obtidas, sempre observando a questão da segurança pessoal tanto dos entrevistadores como dos participantes do estudo.

Para este artigo, a variável dependente foi "Exploração sexual" (não/sim) obtida a partir da pergunta "Alguma vez na vida você já fez sexo em troca de dinheiro, favores ou vantagens?". Como variáveis independentes, foram analisadas as seguintes: sexo (masculino/feminino), idade (10 a $15 / 16$ a 17), vínculo escolar (estuda/nunca estudou ou parou de estudar), mora com a família (não/sim), anos em situação de rua (até 4 anos/5 a 9 anos/10 anos ou mais), horas na rua por dia (até 14 horas/15 horas ou mais), dorme na rua (não/sim); agressão com objeto (não/sim), abuso sexual no contexto familiar (não/sim), relação sexual forçada (não/sim), experimentou crack (não/sim), experimentou droga ilícita (não/sim).

\section{Análise de dados}

Os dados coletados foram tabulados e posteriormente migrados para o software RDSAT 7.1 para que fosse realizada a análise de equilíbrio da amostra. Posteriormente, os dados transferidos para o pacote estatístico Stata IC 13.0, no qual foram conduzidos os procedimentos estatísticos. Foram conduzidas análises univariadas para descrever a amostra e medir a prevalência de exploração sexual. Análises bivariadas foram utilizadas para calcular a prevalência do desfecho de acordo com as categorias de exposição. Análises bruta e ajustada foram realizadas através da regressão de Poisson com ajuste robusto da variância ${ }^{22}$, de acordo com um modelo hierárquico de análise de quatro níveis ${ }^{23}$. As variáveis foram selecionadas através do método backward, ou seja, mantiveram-se no modelo aquelas com valor $\mathrm{p} \leq 0,2$, como estratégia de controle de confundidores. Foi adotado um nível de significância de 5\% para testes bicaudais.

\section{Aspectos éticos}

As crianças e adolescentes foram convidadas a participar do estudo, tendo sido explicados os objetivos e o processo de recrutamento através do RDS. Por se tratar de indivíduos com idade inferior a 18 anos, com pouco ou nenhum contato com suas famílias e responsáveis legais, a anuência dos participantes foi obtida de forma verbal, através do Assentimento Livre e Esclarecido dos participantes ${ }^{24}$. Como a obtenção do assentimento não elimina a necessidade do consentimento do responsável, conforme recomenda Resolução no 510/2016 do Conselho Nacional de Saúde ${ }^{25}$, foi solicitada - e concedida - permissão para as Varas da Infância e da Juventude das cidades de Porto Alegre e de Rio Grande para acesso ao grupo que vive em situação de rua, realização das entrevistas e a utilização de cupons. O projeto de pesquisa foi aprovado pelo Comitê de Ética em Pesquisa da FURG. O estudo maior contou com o financiamento do Programa Nacional de DST/ HIV-AIDS do Ministério da Saúde.

\section{Resultados}

Participaram do estudo 307 crianças, adolescentes e jovens em situação de rua, sendo 204 entrevistados em Porto Alegre e 103 em Rio Grande. Neste estudo realizou-se um recorte com foco na variável exploração sexual. Para tanto a amostra do presente artigo foi composta apenas por indivíduos com idade até 18 anos incompletos, totalizando 186 crianças e adolescentes. A amostra final deste estudo atingiu o equilíbrio, de acordo com o método RDS, segundo parâmetros estabelecidos pelo software RDSat.

A Tabela 1 apresenta a descrição da amostra, a qual é formada majoritariamente por indivíduos com idades entre 10 e 15 anos (65\%) e do sexo masculino $(80,1 \%)$. Dos entrevistados, $31,8 \%$ nunca estudaram ou pararam de estudar, $30,1 \%$ da amostra não morava com a família e, quase um terço, estava na rua por cinco anos ou mais $(32,8 \%)$. Um quarto da amostra relatou dormir na rua e $16,7 \%$ reportaram passar 15 horas ou mais na rua. Quase metade sofreu agressão com objeto (43,6\%), 3,2\% já sofreram assédio sexual no ambiente familiar e $7 \%$ dos participantes já tiveram relação sexual forçada. Mais da metade experimentou droga ilícita pelo menos uma vez na vida e $27,4 \%$ da amostra já experimentaram crack. A prevalência de exploração sexual identificada nesta investigação foi de $8,0 \%$.

A Tabela 2 mostra a prevalência de exploração sexual de acordo com as categorias deste estudo, bem como os resultados das análises bruta e ajustada. A maior prevalência de exploração sexual encontra-se entre aqueles que sofreram assédio sexual no ambiente familiar $(50,0 \%)$ e a menor prevalência entre aqueles que possuíam vínculo escolar $(0,8 \%)$. Na análise bruta (qui-quadrado) foi observado que ser mais velho, não ter vínculo escolar, não morar com a família, passar mais 
Tabela 1. Descrição da amostra de crianças e adolescentes em situação de rua, com idades entre 10 e 17 anos $(\mathrm{N}=186)$. Porto Alegre e Rio Grande, Rio Grande do Sul, Brasil.

\begin{tabular}{|c|c|c|}
\hline Variável & $\begin{array}{c}\text { Participantes } \\
\text { (n) }\end{array}$ & $\begin{array}{c}\text { Percentual } \\
(\%)\end{array}$ \\
\hline \multicolumn{3}{|l|}{ Sexo } \\
\hline Masculino & 149 & 80,1 \\
\hline Feminino & 37 & 19,9 \\
\hline \multicolumn{3}{|l|}{ Idade } \\
\hline 10-15 anos & 121 & 65,0 \\
\hline 16-17 anos & 65 & 35,0 \\
\hline \multicolumn{3}{|l|}{ Vínculo escolar } \\
\hline Estuda & 127 & 68,2 \\
\hline $\begin{array}{l}\text { Nunca estudou ou } \\
\text { parou }\end{array}$ & 59 & 31,8 \\
\hline \multicolumn{3}{|l|}{ Mora com a família } \\
\hline $\operatorname{Sim}$ & 130 & 69,9 \\
\hline Não & 56 & 30,1 \\
\hline \multicolumn{3}{|l|}{$\begin{array}{l}\text { Anos em situação de } \\
\text { rua }\end{array}$} \\
\hline Até 4 anos & 125 & 67,2 \\
\hline 5 anos ou mais & 61 & 32,8 \\
\hline \multicolumn{3}{|l|}{ Horas na rua por dia } \\
\hline Até 14 horas & 155 & 83,3 \\
\hline 15 horas ou mais & 31 & 16,7 \\
\hline \multicolumn{3}{|l|}{ Dorme na rua } \\
\hline Não & 141 & 75,8 \\
\hline $\operatorname{Sim}$ & 45 & 24,1 \\
\hline \multicolumn{3}{|l|}{ Agressão com objeto } \\
\hline Não & 105 & 56,4 \\
\hline $\operatorname{Sim}$ & 81 & 43,6 \\
\hline \multicolumn{3}{|l|}{$\begin{array}{l}\text { Abuso sexual no } \\
\text { contexto familiar }\end{array}$} \\
\hline Não & 180 & 96,8 \\
\hline $\operatorname{Sim}$ & 6 & 3,2 \\
\hline \multicolumn{3}{|l|}{ Relação sexual forçada } \\
\hline Não & 173 & 93,0 \\
\hline Sim & 13 & 7,0 \\
\hline \multicolumn{3}{|l|}{ Experimentou crack } \\
\hline Não & 135 & 72,6 \\
\hline Sim & 51 & 27,4 \\
\hline \multicolumn{3}{|l|}{$\begin{array}{l}\text { Experimentou droga } \\
\text { ilícita }\end{array}$} \\
\hline Não & 84 & 45,0 \\
\hline $\operatorname{Sim}$ & 102 & 55,0 \\
\hline \multicolumn{3}{|l|}{ Exploração sexual } \\
\hline Não & 172 & 92,0 \\
\hline Sim & 14 & 8,0 \\
\hline
\end{tabular}

horas por dia na rua, dormir na rua, ter sofrido agressão com objeto, ter experiência de assédio sexual no ambiente familiar, ter sofrido relação sexual forçada e ter experimentado drogas ilícitas foram características associadas à exploração sexual. Entretanto, após a análise ajustada, permaneceram independentemente associadas ao desfecho ter idade entre 16 e 17 anos $(\mathrm{RP}=3,35$; IC95\% 1,17-9,61), não ter vínculo escolar $(\mathrm{RP}=16,29$; IC95\% 1,82-145,70) e ter sofrido relação sexual forçada (RP=2,7; IC95\% 1,07-6,86).

\section{Discussão}

Esse estudo teve como objetivo investigar a prevalência de exploração sexual entre crianças e adolescentes em situação de rua e as características associadas esse desfecho. Conforme foi apresentado, identificou-se que $8 \%$ dos participantes da pesquisa já tiveram experiência de exploração sexual. Indivíduos mais velhos, sem vínculo escolar e que sofreram alguma relação sexual forçada tiveram maior probabilidade de terem sido sexualmente explorados. A prevalência de exploração sexual neste estudo foi elevada quando comparada a estudos com amostras gerais de adolescentes. Pesquisas realizadas nos Estados Unidos e no Brasil encontraram frequências de 3,5\% ${ }^{26} \mathrm{e}$ $6,0 \%{ }^{27}$, respectivamente. Contudo, investigações com amostras de pessoas em situação de rua encontraram prevalências superiores, variando entre $18,2 \%{ }^{28}$ até $40,0 \%{ }^{29}$. Estimar a prevalência de um fato de natureza criminosa, que ocorre com um grupo populacional de difícil acesso e que reluta em informar sua real situação é um desafio. Estudos sugerem que as taxas de exploração sexual contra crianças e adolescentes em situação de rua sejam muito maiores do que aquelas identificadas nas coletas de dados ${ }^{30}$, justamente pelas restrições impostas pela temática, acesso e métodos empregados.

Crianças e adolescentes em situação de rua são explorados sexualmente de forma recorrente, diante da necessidade de dinheiro, drogas, abrigo ou proteção de adultos que oferecem acesso a necessidades específicas desse grupo em troca de sexo $^{3,31,32}$. Aqueles com idades entre 16 e 17 anos tiveram maior probabilidade de ter sofrido exploração sexual. Resultado semelhante foi encontrado em um estudo canadense, no qual a média de idade entre pessoas em situação de rua que já tinham sido exploradas e receberam dinheiro por sexo foi maior do que entre aqueles que não tinham sido exploradas ${ }^{33}$. Em um estudo 
Tabela 2. Prevalências por categorias e razões de prevalências bruta e ajustada da associação entre exploração sexual e variáveis independentes. Análise multivariável conduzida através da regressão de Poisson com ajuste robusto da variância, de acordo com modelo hierárquico de análise de quatro níveis. Porto Alegre e Rio Grande, Rio Grande do Sul, Brasil.

\begin{tabular}{|c|c|c|c|c|}
\hline Nível & Variáveis & $\begin{array}{l}\text { Exploração } \\
\text { Sexual (\%) }\end{array}$ & $\begin{array}{l}\text { Análise bruta } \\
\text { RP (IC95\%) }\end{array}$ & $\begin{array}{c}\text { Análise ajustada } \\
\text { RP (IC95\%) }\end{array}$ \\
\hline \multirow[t]{6}{*}{$1^{\circ}$} & Sexo & & $\mathrm{p}=0,127$ & $\mathrm{p}=0,304$ \\
\hline & Masculino & 6,0 & 1 & 1 \\
\hline & Feminino & 13,5 & $2,24(0,79-6,30)$ & $1,78(0,59-5,34)$ \\
\hline & Idade & & $\mathrm{p}=0,024$ & $\mathrm{p}=0,024$ \\
\hline & $10-15$ anos & 4,1 & 1 & 1 \\
\hline & 16-17 anos & 13,9 & $3,35(1,17-9,61)$ & $3,35(1,17-9,61)$ \\
\hline \multirow[t]{15}{*}{$2^{\circ}$} & Vínculo escolar & & $\mathrm{p}<0,001$ & $\mathrm{p}=0,013$ \\
\hline & Estuda & 0,8 & 1 & 1 \\
\hline & Nunca estudou/parou & 22,0 & $27,98(3,72-210,06)$ & $16,29(1,82-145,70)$ \\
\hline & Mora com a família & & $\mathrm{p}=0,008$ & $\mathrm{p}=0,820$ \\
\hline & Sim & 3,9 & 1 & 1 \\
\hline & Não & 16,1 & $4,18(1,46-11,94)$ & $0,84(0,20-3,63)$ \\
\hline & Anos em situação de rua & & $\mathrm{p}=0,162$ & $\mathrm{p}=0,743$ \\
\hline & Até 4 anos & 5,6 & 1 & 1 \\
\hline & 5 anos ou mais & 11,5 & $2,05(0,75-5,60)$ & $1,17(0,45-3,06)$ \\
\hline & Horas na rua por dia & & $\mathrm{p}=0,003$ & $\mathrm{p}=0,113$ \\
\hline & Até 14 horas & 3,9 & 1 & 1 \\
\hline & 15 horas ou mais & 25,8 & $6,67(2,48-17,91)$ & $2,21(0,83-5,88)$ \\
\hline & Dorme na rua & & $\mathrm{p}=0,001$ & $\mathrm{p}=0,960$ \\
\hline & Não & 3,6 & 1 & 1 \\
\hline & Sim & 20,0 & $5,64(1,99-16,01)$ & $0,95(0,13-7,19)$ \\
\hline \multirow[t]{9}{*}{$3^{\circ}$} & Agressão com objeto & & $\mathrm{p}=0,014$ & $\mathrm{p}=0,162$ \\
\hline & Não & 2,9 & 1 & 1 \\
\hline & $\operatorname{Sim}$ & 13,6 & $4,75(1,37-16,53)$ & $2,38(0,71-8,00)$ \\
\hline & Abuso sexual no contexto familiar & & $\mathrm{p}=0,012$ & $\mathrm{p}=0,380$ \\
\hline & Não & 6,1 & 1 & 1 \\
\hline & $\operatorname{Sim}$ & 50,0 & $8,18(3,05-21,94)$ & $1,63(0,55-4,90)$ \\
\hline & Relação sexual forçada & & $\mathrm{p}=0,006$ & $\mathrm{p}=0,035$ \\
\hline & Não & 5,2 & 1 & 1 \\
\hline & Sim & 38,5 & $7,39(2,89-18,91)$ & $2,71(1,07-6,86)$ \\
\hline \multirow[t]{6}{*}{$4^{\circ}$} & Experimentou crack & & $\mathrm{p}<0,001$ & $\mathrm{p}=0,350$ \\
\hline & Não & 2,2 & 1 & 1 \\
\hline & $\operatorname{Sim}$ & 21,6 & $9,71(2,81-33,49)$ & $1,82(0,52-6,43)$ \\
\hline & Experimentou droga ilícita & & $\mathrm{p}=0,033$ & $\mathrm{p}=0,603$ \\
\hline & Não & 2,4 & 1 & 1 \\
\hline & Sim & 11,8 & $4,94(1,13-21,55)$ & $0,57(0,07-4,76)$ \\
\hline
\end{tabular}

\%=Prevalência de exploração sexual por categoria de exposição; RP=Razão de prevalências; IC95\%=Intervalo de confiança de $95 \%$.

Fonte: Elaborado pelos autores.

feito no Paquistão, participantes mais velhos (1519 anos) tiveram probabilidade $85 \%$ maior de ter sido explorado e ter recebido dinheiro ou favores em troca de sexo do que os mais novos, apesar dessa associação não ter se mantido após análise de regressão ${ }^{29}$. Uma hipótese para esse resultado é de que adolescentes mais velhos já tenham características sexuais secundárias mais desenvolvidas e que seus corpos possam aproximar-se dos padrões socialmente estabelecidos, aumentando o risco de serem alvos de violência sexu$\mathrm{al}^{34}$. Por exemplo, em um estudo conduzido com caminhoneiros brasileiros, foi identificado que o principal motivo pelo qual homens preferem fa- 
zer sexo com crianças e adolescentes foi "ter mais excitação e prazer”. No entender dos participantes dessa pesquisa, esse prazer se dá em função do padrão de beleza da juventude, hegemônico na sociedade e na mídia² ${ }^{2}$ É possível também que crianças menores tenham maior dificuldade de compreender a situação de exposição a situação abusiva $^{35}$ e não a identifiquem como exploração.

Participantes que sofreram relação sexual forçada apresentaram maior probabilidade de serem vítimas de exploração sexual. Uma das hipóteses para este resultado é de que as violências sexuais sofridas possam produzir prejuízos à sexualidade da vítima. Existem evidências de que pessoas com histórico de abuso sexual na infância estão mais propensas a desenvolver problemas médicos, psicológicos, comportamentais e sexuais ${ }^{36}$ e mais expostas ao risco de exploração sexual ${ }^{37}$. A vida das crianças e adolescentes que usam as ruas brasileiras como contexto de moradia e/ou trabalho é marcada por violações que, por vezes, iniciam ainda dentro de casa ${ }^{12}$, sendo comum o histórico de abuso sexual na infância ${ }^{3,38}$. No entanto, essa não é uma exclusividade do Brasil. Uma meta-análise com dados de 8 países encontrou que adolescentes com histórico de abuso sexual estão 2,5 vezes mais suscetíveis a serem explorados sexualmen$\mathrm{te}^{39}$. Em uma pesquisa qualitativa com mulheres que foram vítimas de abuso sexual na infância, foi identificado que conduta hiperssexualizada e prostituição eram algumas das principais repercussões do abuso sofrido ${ }^{40}$. Pesquisas qualitativas com adolescentes exploradas ainda sugerem que a erotização do corpo e corresponsabilização pela situação abusiva sofrida são fatores colaboradores para novas vitimizações ${ }^{3,38}$.

Estar fora da escola também é um fator de risco. Um terço dos participantes não possui vínculo escolar, taxa quatro vezes superior à população geral $^{41}$. É possível que uma parcela ainda maior desta população não compareça na escola, conforme identificado em outra investigação com crianças e adolescentes em situação de rua que apontou que $63,3 \%$ não frequentavam escola ${ }^{42}$. Crianças e adolescentes que abandonaram os estudos ou que nunca estudaram tiveram probabilidade 16 vezes maior de terem sido sexualmente exploradas do que aquelas com vínculo escolar, sendo esta variável a principal preditora de exploração sexual em nosso modelo de análise.

Em um estudo longitudinal realizado com mais de 20.000 adolescentes observou-se que os princípios de conexão com a escola apresentam um efeito protetor na exploração sexual comer$\mathrm{cial}^{43}$. Percebe-se que a escola contribui signifi- cativamente para a proteção e expectativas em relação ao futuro dessas crianças e adolescentes, pois diminui a exposição à violência e condições de precariedade e vulnerabilidade da rua colaborando para o desenvolvimento ${ }^{44}$. Crianças e adolescentes em situação de vulnerabilidade estão mais expostos à pobreza, à famílias marcadas por ciclos de violência, abuso de álcool e outras drogas e abuso sexual. Nestes casos, estar fora de escola e/ou estar em situação de rua são fatores que contribuem para maximizar estes riscos ${ }^{45}$.

Os resultados desse estudo devem ser interpretados sob a luz de suas limitações e seus pontos fortes. O delineamento transversal pode ser considerado uma limitação, pois trata-se de um tipo de estudo que não permite o estabelecimento de temporalidade, consequentemente, os resultados estão sujeitos ao viés de causalidade reversa. Todavia, por se tratar de uma população volátil, investigações longitudinais podem se tornar inexequíveis, fazendo dos estudos transversais uma ferramenta importante na avaliação da saúde desse subgrupo. Outra limitação é o viés de não-aceitação (ou falsa resposta), pelo fato de o estudo ter realizado perguntas diretas sobre comportamentos ilegais e estigmatizados. Mesmo tendo sido adotados procedimentos éticos e técnicos padronizados, alguns entrevistados podem ter se sentido desconfortáveis em responder algumas perguntas.

Um diferencial metodológico dessa investigação foi o uso da técnica de amostragem RDS, que permite acessar populações de difícil acesso (como as pessoas em situação de rua) de forma a produzir uma amostra final probabilística. $\mathrm{O}$ RDS tem sido recomendado para o desenvolvimento de estudos sobre exploração sexual justamente por alcançar grupos que os métodos tradicionais não seriam capazes ${ }^{46}$. Embora existam orientações para o cuidado e verificação dos critérios de inclusão dos convidados trazidos pelas sementes ${ }^{47}$, quando estão envolvidos aqueles que estão em situação de rua a atenção da equipe de pesquisa precisa ser aumentada. Convidados podem ser trazidos sem que haja qualquer preocupação com os critérios de inclusão explicitados, o mesmo convidado pode ser trazido mais de uma vez, o cupom-convite distribuído pode ser extraviado ou furtado devido a própria dificuldade de privacidade e cuidado com objetos pessoais ou, ainda, o cupom-convite pode servir como uma moeda de troca entre o grupo. $\mathrm{O}$ uso de linguagem simples, vestimenta adequada e conhecimento técnico, teórico e ético favorecem a vinculação com a população. Equipes bem treinadas podem minimizar esses problemas. 


\section{Considerações finais}

Conclui-se neste estudo que $8 \%$ das crianças e adolescentes investigadas foram exploradas sexualmente, principalmente aquelas mais velhas e que já tinham tido sido violadas anteriormente através de relação sexual forçada. Contudo, a principal variável preditora do nosso modelo foi o vínculo escolar. Participantes que não frequentavam a escola tiveram probabilidade 16 vezes maior de ter sido sexualmente explorados, o que sugere ser este um importante ambiente de proteção. Portanto, recomenda-se o fortalecimento de intervenções que venham a priorizar os vínculos de crianças e adolescentes em situação de rua com a escola. Embora escola e rua pareçam dissonantes e estejam frequentemente atreladas ao fracasso escolar, as experiências da escola aberta podem ser interessantes, já que ofertam um sistema de ensino flexível e adaptado as necessidades de quem usa o contexto da rua como moradia e/ ou trabalho ${ }^{48}$. Sugere-se aos profissionais e às autoridades envolvidas, que sejam feitas avaliações frequentes sobre as relações entre as crianças e adolescentes em situação de rua e a escola e outros contextos educativos não-formais. É possível que ambientes como albergues, centros de referência especializados para esta população e/ou eventos de caridade, possam ser ambientes oportunos para esta avaliação. Serviços das políticas de assistência social, saúde, defesa de direitos e educação precisam estar integrados. Crianças e adolescentes em situação de rua e explorados sexualmente são uma população oculta, em constante movimento e com riscos significativos. São inúmeros os crimes cometidos contra elas que ainda permanecem velados pelas violações de direito acometidas dentro do próprio lar, pelas iniquidades sociais e pela própria sociedade. Estudos que avaliem os mecanismos pelos quais a escola protege as crianças e os adolescentes em situação de rua da exploração sexual podem auxiliar no entendimento deste fenômeno e, consequentemente, otimizar futuras políticas e intervenções integradas em saúde pública voltadas a essa população.

\section{Colaboradores}

LM Demenech, SS Paludo e L Neiva-Silva contribuíram com a concepção e delineamento do artigo, na análise e interpretação dos dados e na escrita e revisão crítica do manuscrito. PS Silva, AMN Paiva e F Fontes contribuíram na análise e interpretação dos dados e na escrita do manuscrito. Todos os autores aprovaram a versão final apresentada nesta submissão. 


\section{Referências}

1. Deslandes SF, Campos DS. A ótica dos conselheiros tutelares sobre a ação da rede para a garantia da proteção integral a crianças e adolescentes em situação de violência sexual. Cien Saude Colet 2015; 20(7):21732182.

2. Morais NA, Cerqueira-Santos E, Moura AS, Vaz M, Koller SH. Exploração sexual comercial de crianças e adolescentes: um estudo com caminhoneiros brasileiros. Psic Teor Pesq 2007; 23(3):263-271.

3. Vega LBS, Paludo SS. Exploração sexual e rede de proteção na perspectiva da vítima. Arq Bras Psicol 2015; 67(2):47-60.

4. Paludo SS, Ferreira LS, Vega LBS. Dilemas e desafios éticos na pesquisa sobre exploração sexual. Rev $S P A-$ GESP 2017; 18(2):115-128.

5. Lowenkron L. Abuso sexual infantil, exploração sexual de crianças, pedofilia: diferentes nomes, diferentes problemas? Sex Salud Soc (Rio J) 2010; 5:9-29.

6. Alberto MFP, Silva ACS, Gomes VC, Santana RVM, Soares AM. Os agentes sociais da rede de proteção e atendimento no enfrentamento da exploração sexual comercial. Psicol-Reflex Crit 2012; 25(1):130-138.

7. Salazar-Muñoz M, Vega-Eeles D. Características psicosociales asociadas a mujeres adolescentes víctimas de explotación sexual comercial, que ingresaron a un centro de atención en la región de Los Lagos, Chile. Rev Crim 2017; 59(3):193-214.

8. International Labour Organization (ILO). Every child counts: new global estimates on child labour. Geneva: ILO; 2002.

9. United Nations Children's Fund (UNICEF). The state of the world's children 2006: Excluded and invisible. New York: UNICEF; 2006.

10. Childhood Brasil. Relatório de atividades 2018. São Paulo: Childhood Brasil; 2019.

11. Simon I, Galera ES. Exploração sexual comercial de crianças e adolescentes. Rev Juridic (FURB) 2017; $1(1): 115-140$.

12. Rizzini I, Couto RMB. População infantil e adolescente nas ruas: Principais temas de pesquisa no Brasil. Civitas 2019; 19(1):105-122.

13. Gontijo DT, Medeiros M. Crianças e adolescentes em situação de rua: contribuições para a compreensão dos processos de vulnerabilidade e desfiliação social. Cien Saude Colet 2009; 14(2):467-475.

14. Barbiani R. Violação de direitos de crianças e adolescentes no Brasil: interfaces com a política de saúde. Saude Debate 2016; 40(109):200-211.

15. Brasil. Lei $n^{\circ} 8.069$, de 13 de julho de 1990. Dispõe sobre o Estatuto da Criança e do Adolescente e dá outras providências. Diário Oficial da União 1990; 16 jul.

16. Moura YG, Sanchez ZM, Opaleye ES, Neiva-Silva L, Koller SH, Noto AR. Drug use among street children and adolescents: what helps? Cad Saude Publica 2012; 28(7):1371-1380.

17. Neiva-Silva L, Koller SH. A rua como contexto de desenvolvimento. In: Lordelo ER, Carvalho AMA, Koller $\mathrm{SH}$, editores. Infância brasileira e contextos de desenvolvimento. São Paulo: Casa do Psicólogo; 2002.
18. Noto AR, Galduróz JCF, Nappo SA, Fonseca AM, Carlini CMA, Moura YG, Carlini EA. Levantamento nacional sobre o uso de drogas entre crianças e adolescentes em situação de rua nas 27 capitais brasileiras, 2003. São Paulo: Centro Brasileiro sobre Drogas Psicotrópicas; 2004.

19. Heckathorn DD. Respondent-Driven Sampling: A New Approach to the Study of Hidden Populations. Soc Problems 1997; 44(2):174-199.

20. Salganik MJ, Heckathorn DD. Sampling and Estimation in Hidden Populations Using Respondent-Driven Sampling. Sociol Methodol 2004; 34(1):193-240.

21. Carvalho FT, Neiva-Silva L, Ramos MC, Evans J, Koller SH, Piccinini CA, Page-Shafer K. Sexual and drug use risk behaviors among children and youth in street circumstances in Porto Alegre, Brazil. AIDS Behav 2006; 10(Supl. 4):S57-S66.

22. Barros AJ, Hirakata VN. Alternatives for logistic regression in cross-sectional studies: an empirical comparison of models that directly estimate the prevalence ratio. BMC Med Res Methodol 2003; 3:21.

23. Victora CG, Huttly SR, Fuchs SC, Olinto MT. The role of conceptual frameworks in epidemiological analysis: a hierarchical approach. Int J Epidemiol 1997; 26(1):224-227.

24. Morais NA, Lima RFF, Vezedek L, Santana JP, Koller SH. Ética na pesquisa com crianças e adolescentes em situação de rua: considerações a partir da resolução ${ }^{\circ}$ 510/2016. Rev SPAGESP 2017; 18(2):27-42.

25. Brasil. Resolução no 510, de 07 de abril de 2016. Dispõe sobre as normas aplicáveis a pesquisas em Ciências Humanas e Sociais. Diário Oficial da União 2016; 24 maio.

26. Edwards JM, Iritani BJ, Hallfors DD. Prevalence and correlates of exchanging sex for drugs or money among adolescents in the United States. Sex Transm Infect 2006; 82(5):354-358.

27. Teixeira SAM, Taquette SR. Violência e atividade sexual desprotegida em adolescentes menores de 15 anos. Rev Assoc Med Bras 2010; 56:440-446.

28. Owoaje ET, Uchendu OC. Sexual risk behaviour of street youths in south west Nigeria. East Afr J Public Health 2009; 6(3):274-279.

29. Towe VL, ul Hasan S, Zafar ST, Sherman SG. Street life and drug risk behaviors associated with exchanging sex among male street children in Lahore, Pakistan. J Adolesc Health 2009; 44(3):222-228.

30. Barnert E, Iqbal Z, Bruce J, Anoshiravani A, Kolhatkar G, Greenbaum J. Commercial Sexual Exploitation and Sex Trafficking of Children and Adolescents: A Narrative Review. Acad Pediatr 2017; 17(8):825-829.

31. Tyler KA, Johnson KA. Trading sex: voluntary or coerced? The experiences of homeless youth. J Sex Res 2006; 43(3):208-216.

32. Van Leeuwen JM, Hopfer C, Hooks S, White R, Petersen J, Pirkopf J. A snapshot of substance abuse among homeless and runaway youth in Denver, Colorado. $J$ Community Health 2004; 29(3):217-229. 
33. Chettiar J, Shannon K, Wood E, Zhang R, Kerr T. Survival sex work involvement among street-involved youth who use drugs in a Canadian setting. J Public Health (Oxf) 2010; 32(3):322-327.

34. Ssewanyana D, Mwangala PN, Marsh V, Jao I, van Baar A, Newton CR, Abubakar A. Young people's and stakeholders' perspectives of adolescent sexual risk behavior in Kilifi County, Kenya: A qualitative study. $J$ Health Psychol 2018; 23(2):188-205.

35. Pfeiffer L, Salvagni EP. Visão atual do abuso sexual na infância e adolescência. J Pediat 2005; 81(Supl. 5):s197-s204.

36. Maniglio R. The impact of child sexual abuse on health: a systematic review of reviews. Clin Psychol Rev 2009; 29(7):647-657.

37. Varma S, Gillespie S, McCracken C, Greenbaum VJ. Characteristics of child commercial sexual exploitation and sex trafficking victims presenting for medical care in the United States. Child Abuse Negl 2015; 44:98-105.

38. Coutinho MML, Lima RFF, Morais NA. Adolescência, situação de rua e exploração sexual: um estudo de caso. Psicol Teor Prat 2018; 20(3):167-179.

39. De Vries I, Goggin KE. The Impact of Childhood Abuse on the Commercial Sexual Exploitation of Youth: A Systematic Review and Meta-Analysis. Trauma Violence Abuse 2020; 21(5):886-903.

40. Lira MOSC, Rodrigues VP, Rodrigues AD, Couto TM, Gomes NP, Diniz NMF. Abuso sexual na infância e suas repercussões na vida adulta. Texto Contexto Enferm 2017; 26(3):e0080016.

41. Instituto Nacional de Estudos e Pesquisas Educacionais Anísio Teixeira (Inep). Sinopses Estatísticas da Educação Básica. Brasília: Inep; 2018.

42. Elkoussi A, Bakheet S. Volatile substance misuse among street children in Upper Egypt. Subst Use Misuse 2011; 46(Supl. 1):35-39.
43. Kaestle CE. Selling and buying sex: a longitudinal study of risk and protective factors in adolescence. Prev Sci 2012; 13(3):314-322.

44. Ferreira FPM. Crianças e Adolescentes em Situação de Rua e seus Macro Determinantes. Saude Soc 2011; 20(2):338-349.

45. United Nations Children's Fund (UNICEF). The state of the world's children 2012: Children in an urban world. New York: UNICEF; 2012.

46. Gerassi L, Edmond T, Nichols A. Design strategies from sexual exploitation and sex work studies among women and girls: Methodological considerations in a hidden and vulnerable population. Action Res (Lond) 2017; 15(2):161-176.

47. Gile KJ, Handcock MS. Respondent-Driven Sampling: An Assessment of Current Methodology. Sociol Methodol 2010; 40(1):285-327.

48. Schirò EDB, Koller SH, Paludo SS. Educação social para crianças em situação de rua no Brasil. Rev Port Pedagog 2009; 43(2):57-80.

Artigo apresentado em 14/06/2020

Aprovado em 08/09/2020

Versão final apresentada em 10/09/2020

Editores-chefes: Romeu Gomes, Antônio Augusto Moura da Silva 